\title{
Quinoa Peanut Meal Beets Whole Grain Gluten-Free High Protein Vegetable Snacks
}

\author{
Talwinder S Kahlon*, Roberto J Avena-Bustillos and Mei-Chen M Chiu \\ Western Regional Research Center, USA
}

Submission: October 27, 2017; Published: November 17, 2017

*Corresponding author: Talwinder S Kahlon, Western Regional Research Center, USDA-ARS, 800 Buchanan St., Albany, CA 94710, Tel: 510-559-5665; Fax: 510-559-5945; Email: Talwinder.Kahlon@ars.usda.gov

\begin{abstract}
Whole grain gluten-free high protein vegetable snacks were evaluated. The snacks were Quinoa-Peanut Meal-Beets (QPB), QPB-Onion, QPBGarlic and QPB-Ginger. Peanut meal, a high protein low value ingredient, was utilized to increase the protein content of these snacks as well as to add value to this low value agricultural byproduct. Snack dough was prepared using $110-120 \mathrm{ml}$ water for $100 \mathrm{~g}$ as is ingredients. About $20 \mathrm{~g}$ of snack dough was placed on preheated KrumKake Express 839 Baker and cooked for 2 minutes. Seventy seven in-house volunteers judged Color/Appearance to be similar for the snacks tested. Odor/Aroma of QPB-Onion and QPB-Garlic snacks was similar and significantly ( $\mathrm{p} \leq 0.05$ ) higher than QPB and QPB-Ginger snacks. Taste/Flavor and Texture/Mouth feel for QPB-Garlic snacks was significantly better than QPB and QPBGinger snacks. Acceptability of QPB-Onion, QPB-Garlic and QPB snacks was very desirable (78-88\%) and significantly higher than QPB-Ginger $(65 \%)$ snacks. Water activity of all the snacks tested ranged from $0.43-0.47$ suggesting that these snacks were crispy and would have good antimicrobial stability. These snacks were quite fluffy due to their expansion of 2.5-2.8 and 0.60-64\% porosity. The snacks tested were more than $50 \%$ lower in fat as well as in salt than some of the whole grain vegetable snacks available in the market. These snacks could be made in any house hold using table top kitchen appliances and/or in commercial production. The whole grain gluten-free high protein (24-28\%) vegetable healthy nutritious tasty snacks offer choice for vegetarians as well as individuals sensitive to gluten.
\end{abstract}

Keywords: Quinoa; Peanut Meal; Beets; Whole grain; Gluten-free; Snacks

Abbreviations: QPB: Quinoa Peanut Meal-Beets; pb: Bulk Density; pt: true density; Aw: water Activity; DM: Dry Matter

\section{Introduction}

Snacks play an important role in our daily food consumption. In all family and public events snacks are offered. Snacks are preferred over regular meals by the younger generation. The snacks are expected to be tasty, flavorful, healthful and convenient to carry [1]. In school and after school events snacks are available. Healthy snacks should provide good nutrition and support lifelong healthy eating habit. Healthy eating would help in preventing costly and potentially-disabling diseases, such as heart disease, cancer, diabetes, high blood pressure, and obesity. Nutrition Policy and Promotion; Dietary Guidelines for Americans [2], recommends that, at least $1 / 2$ of all the grains eaten should be whole grains. Average intakes of whole grains are less and refined grains are more than recommended levels across all age-sex groups. The Whole Grain Council [3] has documented that eating whole grains instead of refined grains lowers the risk of many chronic diseases. Halnaes et al [4] reported that higher intake of whole grain rye and oats but not wheat lowered the risk of heart disease in men and women. Wheat is the main grain of many snack products, however there is increasing evidence of hyper-sensitivity to gluten in the celiac patients. Lebwohl et al. [5] observed that hypersensitivity to gluten results in eroding of the lining of small intestines. Hypersensitivity to gluten results in delayed growth and many serious health problems. Healthy intestinal lining is required to absorb the needed nutrients. Food \& Drug Administration [6] defines "gluten-free" with less than 20 parts per million gluten. This is the level that can be validly tested and also can be tolerated by most gluten sensitive individual. Hypersensitivity to gluten has been increasing worldwide. Some of the potential reasons could be use of microbial transaminase enzyme as animal protein glue to produce low cost meat patties from meat scraps. Transamination of gluten in the gut could make it hypersensitive. Another reason could be that pollution and toxic environment has altered gut microbiome resulting in loss of gluten immunity. If one family member is gluten sensitive, the whole family has to consume gluten-free foods. As gluten contamination in the kitchen would enlist allergic reaction in celiac patients [7]. Snacks made of gluten-free whole grains would be desirable. Snacks available 
in US are essentially unhealthy and are low protein and low in complex carbohydrates and high in fat and salt. Mother Jones [8] reported that most individuals and especially children and seniors do not meet the recommended intake of whole grains, protein and vegetables. The development of whole grain gluten-free, high protein, vegetable snacks would help to meet such a need. Consumers need to be educated to desire healthy home cooking or health promoting commercially produced foods and snacks. There is a need to develop gluten-free whole grain nutritious tasty snacks. Such snacks could include health promoting spices as well.

\section{Quinoa}

Quinoa (Chenopodium quinoa) is a pseudocereal, round disc shaped grain smaller but similar to sorghum. After harvesting, the quinoa seeds are processed to remove the saponin-containing outer coating. Quinoa cooks like rice and is gluten-free. It is considered perfect food as it contains all nine dietary essential amino acids (histidine, isoleucine, leucine, lysine, methionine, phenylalanine, threonine, tryptophan and valine). Quinoa is a good source of dietary fiber, minerals and unsaturated fatty acids. The Food and Agricultural Organization of the United Nations (FAO), Press Release Note No. 6367, 24 October 2012 [9] officially declared that the year 2013 be recognized as "The International Year of the Quinoa". FAO intended to focus world attention on the role that quinoa can play in providing food security and nutrition, and in the eradication of poverty.

\section{Peanuts}

Peanuts produced in the U.S. are mostly used in food and confection products, but more than 50 percent of the world production is crushed for its oil. World production of peanuts is over 30 million metric tons per year. Nearly 15 million tons of peanuts are used to extract oil resulting in 7.5 million tons of peanut meal. Peanut meal is a low value byproduct mainly used as animal feed. This agricultural byproduct could be used to make high protein nutritious foods and snacks resulting in significant value addition to peanut growers.

\section{Beets}

Beets have been grown for food since ancient times. Both the leaves and the root are edible. Beets most distinguishing characteristic is their bright red pigment, sometimes used as a dye. Beets are also rich source of alkaloid betaine, as well as B-vitamin folate. The betaine and folate together lower blood homocysteine. High levels of homocysteine increase risk of heart disease. In addition it is a moderate source of iron, potassium, vitamin $\mathrm{C}$ and fiber. Beets are rich in nitrates, which the body converts to nitric oxide a compound that relaxes and dilates blood vessels, resulting in better circulation, and possibly lower blood pressure. Kahlon et al. [10] reported that beets bind significantly more bile acids than many other vegetable. Binding bile acids reduces fat absorption with potential to lower cholesterol.

\section{Onion}

Onion (Allium cepa), is a vegetable and is most widely cultivated spice. Slimestad et al. [11] observed that phytonutrients and polyphenols of onion have health promoting potential.

\section{Garlic}

Garlic (Allium sativum) is a widely used spice. Sharp flavor and taste of garlic is due to allicin, a sulfur containing compound. Rahman [12] and Guercio et al. [13] reported that garlic contains several phytonutrients with potential health benefits.

\section{Ginger}

Ginger (Zingiber officinale), is a common spices used in various cuisines and have been associated with various health benefits. Thomson et al. [14] observed that ginger could be used as a cholesterol-lowering, antithrombotic and antiinflammatory agent. Akinyemi et al. [15] and Klein et al. [16] reported that addition of $4 \%$ ginger prevented the effect of hypertension inducing drug in rats.

These snacks would be whole grain, gluten-free, high protein, vegetable, low fat, salt and calories and could be prepared in any home kitchen using counter top appliances and/or by commercial food companies. In the study reported herein, seventy seven in-house volunteers judged snacks containing gluten-free ancient whole grain Quinoa (Q), Peanut meal (P) and Beets (B). The snacks were QPB, QPB-Onion, QPBGarlic and QPB-Ginger.

\section{Materials and Methods}

Whole grain quinoa, raw peanuts, beets (fresh), dry powder (onion, garlic and ginger) were obtained from local grocery markets. Quinoa flour was prepared by using Blendtec Kitchen Mill Model 91 at medium setting (Blendtec Inc., Wichita, Kansas, USA). Peanut meal was obtained by extracting oil using Vevor Oil Press (Joyfay.com, Cleveland, Ohio, USA). Beets were chopped using Mini-Prep Processor (Cuisinart.com, East Windsor, New Jersey, USA).

Ingredients and snacks were analyzed for nitrogen using AOAC method 990.03 [17] by Leco FP628 analyzer (Leco Corporation, St Joseph, MI, USA); crude fat with petroleum ether by accelerated solvent extractor (ASE350, Dionex Corp., Sunnyvale, CA, USA); ash, using AOAC method 942.05 [18]; and moisture, using AOAC method 935.29 [18]. Composition of quinoa flour, peanut meal and beets is given in Table 1. Dough Composition of Ancient Whole Grain Quinoa (Q), Peanut Meal (P) and Beets (B) Snacks is given in Table 2. Snacks dough was prepared by adding $110-120 \mathrm{ml}$ water to $100 \mathrm{~g}$ of as is ingredients. Dough was set at room temperature for $30 \mathrm{~min}$. The levels of garlic, onion and ginger were decided by the consensus of laboratory personnel. Snacks were cooked in a 1050 Watt, Chef's Choice KrumKake Express 839 (Edge Craft 
Corporation, Avondale, PA, USA). KrumKake Express with heat control dial settings 1- 6 was heated at setting 4 till green ready light turned on. Baking temperature ranged from $185-208^{\circ} \mathrm{C}$ as measured by Anritsu Meter AP-710 (Anritsumeter Co., Tokyo, Japan). Cooking surface was lightly treated with non-stick cooking spray. $20 \mathrm{~g}$ snack dough was placed on the center of heated KrumKake Express baker and cooked for $2 \mathrm{~min}$. Figure 1 shows four kinds of gluten-free ancient whole grain QuinoaPeanut meal-Beets cooked snacks. Cooked snacks were cooled to room temperature then vacuum heat sealed in plastic bags by Food Saver 2200 Series (Foodsaver.com, Boca Raton, FL, USA).

Table 1: Composition of Quinoa, Peanut meal and Beets, dry matter basis \%.

\begin{tabular}{|c|c|c|c|c|c|}
\hline Ingredients & Protein & Fat & Minerals & Carbohydrate & DM \\
\hline Quinoa & $16.46 \pm 0.14$ & $7.00 \pm 0.20$ & $3.38 \pm 0.01$ & $62.41 \pm 0.15$ & $89.25 \pm 0.03$ \\
\hline Peanut meal & $59.69 \pm 0.50$ & $15.96 \pm 0.15$ & $3.94 \pm 0.05$ & $14.31 \pm 0.25$ & $93.90 \pm 0.02$ \\
\hline Beets & $1.38 \pm 0.02$ & $0.30 \pm 0.00$ & $0.83 \pm 0.01$ & $8.68 \pm 0.01$ & $11.19 \pm 0.07$ \\
\hline
\end{tabular}

Nitrogen to protein factors used was 6.25. Dry matter, DM.

Carbohydrate $=[$ Dry matter $-($ Protein + Fat + Ash $)]$. Samples were analyzed in triplicates.

Values are Mean \pm SEM.

Table 2: Dough Composition of Ancient Whole Grain Gluten-free Quinoa (Q), Peanut meal (P) and Beets (B) Snacks, as is \%.

\begin{tabular}{|c|c|c|c|c|c|c|c|c|}
\hline Snack & $\mathbf{Q}$ & $\mathbf{P}$ & B & Salt & Garlic & Onion & Ginger & Water \\
\hline QPB & 24.7 & 23.8 & 49.4 & 1.2 & --- & --- & --- & $110 \mathrm{ml}$ \\
\hline QPB-Onion & 24 & 24 & 48 & 1.2 & $--\cdot$ & 2.9 & --- & $120 \mathrm{ml}$ \\
\hline QPB-Garlic & 23.7 & 23.7 & 47.4 & 1.2 & 3.8 & --- & --- & $120 \mathrm{ml}$ \\
\hline QPB-Ginger & 24 & 24 & 48 & 1.2 & --- & --- & 2.9 & $120 \mathrm{ml}$ \\
\hline
\end{tabular}

Level of Garlic, Onion and Ginger was decided by consensus of laboratory personnel.

Dough was set at room temperature for $30 \mathrm{~min}$.
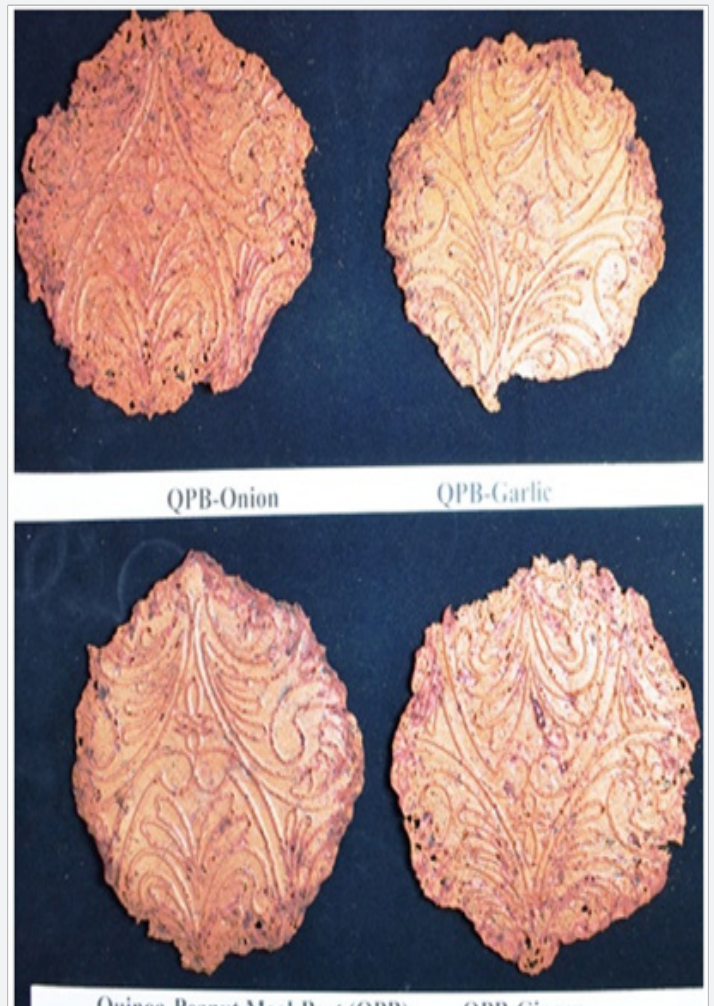

Quinoa-Peanut Meal-Beet (QPB) QPB-Ginger

Figure 1: Bottom left: Quinoa-Peanut Meal-Beets (QPB), Top left: QPB-Onion, Top right: QPB-Garlic, Bottom right: QPBGinger, cooked snacks.

\section{Sensory evaluation of snacks}

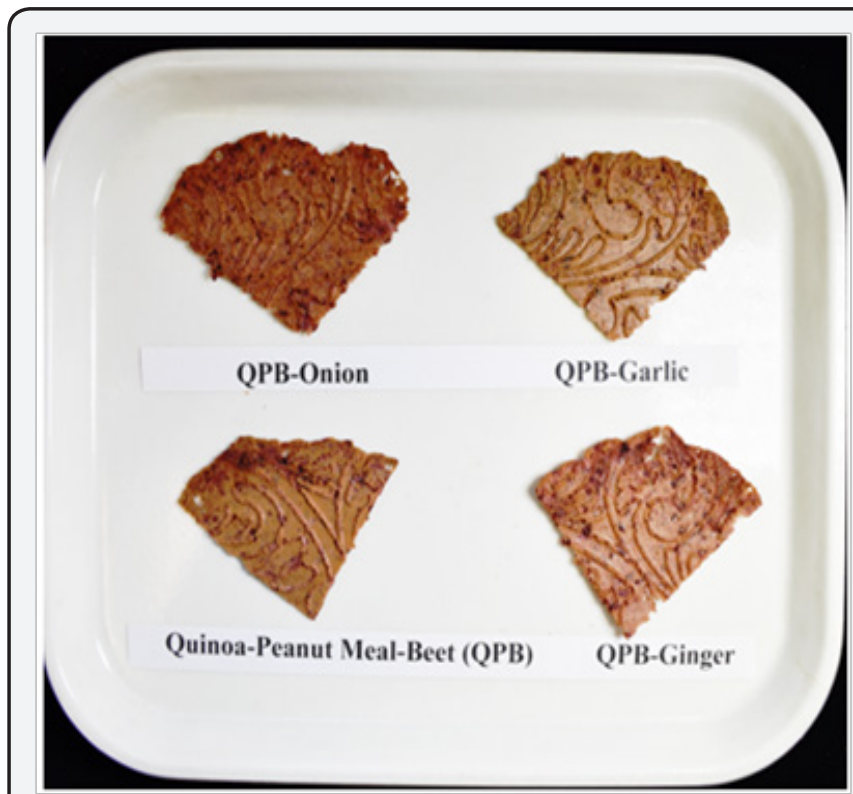

Figure 2: Four kinds of Quinoa whole grains high protein Vegetable snacks as offered for sensory evaluation to the taster, Bottom left: Quinoa-Peanut meal-Beets (QPB); Top left: QPBOnion; Top right: QPB-Garlic; Bottom right: QPB-Ginger.

Vacuum packed cooked snacks were opened and cut into four pieces with a pizza cutter. All four kinds of snacks were placed in an eight inch by ten inch plate labeled QunioaPeanut meal-Beets (QPB)-Onion, QPB-Garlic, QPB, QPB- 
Ginger. Seventy seven in-house volunteers tasted the snacks in four sensory evaluation booths under white light for Color/ Appearance, Odor/Aroma, Taste/Flavor, Texture/Mouth-feel and Acceptance. Snacks were evaluated individually and not for relative rating. The sensory parameters were evaluated on a scale of 1-5 (Like very much=5, like slightly=4, neutral=3, dislike slightly=2 and dislike very much=1); Acceptance was evaluated on a scale of 1-2 (Acceptable=2 and unacceptable=1). For sensory evaluation snacks presented were appropriately labeled. Quinoa whole grains high protein Vegetable snacks as offered for sensory evaluation to the tasters (Figure 2). Bottom left: Quinoa-Peanut meal-Beets (QPB); Top left: QPBOnion; Top right: QPB-Garlic; Bottom right: QPB-Ginger.

Water Activity: Water activity (Aw) of Quinoa-Peanut meal-Beets gluten-free whole grains snacks was measured at $25.01 \pm 0.02{ }^{\circ} \mathrm{C}$ in triplicate using an Aqua Lab 4TE dew point water activity meter (Decagon Devices, Inc., Pullman, WA, USA).

Density: The bulk density ( $\rho$ b) of QPB gluten-free whole grain snacks was measured by Ottawa Sand volume displacement of $10 \mathrm{~g}$ sample in triplicate after shaking in a jar of $202 \mathrm{ml}$ volume. The first reading was taken after shaking for $15 \mathrm{~min}$. subsequent two readings were taken after additional shaking for $5 \mathrm{~min}$ each time. True density ( $\rho \mathrm{t})$ was determined five times using gas displacement pycnometer AccuPycII1340 (Micromeritics Instrument Co., Norcross, GA, USA) at 21.4 \pm 0.4 ${ }^{\circ} \mathrm{C}$. Samples of each snack were dried in triplicate at room temperature for $15 \mathrm{hr}$ at $0 \%$ relative humidity in vacuum Table 3: Results of Taste Panel of Quinoa-Peanut meal-Beets (QPB), desiccators with anhydrous calcium sulfate (W.A. Hammond Drierite, Xenia, OH, USA). After drying, small pieces of samples were compressed into a density measuring cylinder of the pycnometer and five true density values were recorded.

\section{Statistical Analysis}

Data were analyzed with Mini tab software (version14.12.0, Minitab inc., State College, PA, USA) using one way analysis of variance and Tuckey's multiple comparison tests with $(\mathrm{p} \leq 0.05)$ was considered the criterion of significance.

\section{Results and Discussion}

Color/Appearance of Quinoa-Peanut meal-Beets (QPB), QPB-Onion, QPB-Garlic and QPB-Ginger snacks was judged to be similar by 77 in-house volunteer tasters Table 3. Data suggest that intense red color of beets imparted similar Color/ Appearance of the four kinds of gluten-free high protein vegetable snacks tested. Odor/Aroma of QPB-Onion and QPBGarlic snacks was similar and significantly $(\mathrm{p} \leq 0.05)$ higher than QPB and QPB-Ginger snacks. Taste/Flavor of the QPBOnion snacks was significantly higher than QPB-Ginger snacks. Taste/Flavor and Texture/Mouth feel of the QPB-Garlic snacks was significantly better than QPB and QPB-Ginger snacks. Acceptability of QPB, QPB-Onion and QPB-Garlic snacks was similar and significantly higher than QPB-Garlic snacks. Data suggest that tasters did not prefer snacks that contained ginger in several sensory parameters. Consensus laboratory personnel for level of ginger used was higher than preference of diverse volunteer 77 tasters.

\begin{tabular}{|c|c|c|c|c|c|}
\hline Snack & Color Appearance & Odor Aroma & Taste Flavor & Texture Mouth feel & Acceptance \\
\hline QPB & $3.82 \pm 0.11^{\mathrm{a}}$ & $3.61 \pm 0.09^{\mathrm{b}}$ & $3.46 \pm 0.12 \mathrm{~b}^{\mathrm{c}}$ & $3.73 \pm 0.13^{\mathrm{b}}$ & $1.78 \pm 0.05^{\mathrm{a}}$ \\
\hline QPB-Onion & $3.99 \pm 0.11^{\mathrm{a}}$ & $4.04 \pm 0.11^{\mathrm{a}}$ & $3.75 \pm 0.13^{\mathrm{ab}}$ & $4.01 \pm 0.10^{\mathrm{ab}}$ & $1.88 \pm 0.04^{\mathrm{a}}$ \\
\hline QPB-Garlic & $3.83 \pm 0.10^{\mathrm{a}}$ & $3.97 \pm 0.11^{\mathrm{a}}$ & $3.97 \pm 0.13^{\mathrm{a}}$ & $4.19 \pm 0.10^{\mathrm{a}}$ & $1.86 \pm 0.04^{\mathrm{a}}$ \\
\hline QPB-Ginger & $3.95 \pm 0.10^{\mathrm{a}}$ & $3.45 \pm 0.09^{\mathrm{b}}$ & $3.12 \pm 0.13^{\mathrm{c}}$ & $3.81 \pm 0.12^{\mathrm{b}}$ & $1.65 \pm 0.05^{\mathrm{b}}$ \\
\hline
\end{tabular}

aValues (mean \pm SEM) within columns with different superscript letters differ significantly $(p \leq 0.05), n=77$.

'Sensory evaluation parameters were on a scale of 1-5 (Like very much $=5$, like slightly $=4$, neutral $=3$, dislike slightly $=2$ and dislike very much $=1$ ); Acceptance was on scale of 1-2 (Acceptable $=2$ and unacceptable $=1$ ).

Desirability index for sensory parameters was calculated as percent of tasters judged \{like very much + like slightly $+1 / 2$ (neutral)\} Kahlon et al. [19,20]. Desirability index for QPB, QPB-Onion, QPB-Garlic and QPB-Ginger snacks was for Color/Appearance 78\%, 82\%, 79\% and $82 \%$; for Odor/Aroma $74 \%, 83 \%, 84 \%$ and $69 \%$; for Taste/Flavor $68 \%, 77 \%, 81 \%$ and $52 \%$; for Texture/mouth feel $71 \%, 85 \%, 88 \%$ and $77 \%$ respectively. Desirability index for all the sensory parameters were 68-88\% except for Taste/Flavor for QPB-Ginger snacks as $52 \%$. Data suggest that except for QPB-Ginger the other three kinds snacks tested were preferred by the in-house voluntary tasters. Similar 53-88\% acceptability of gluten-free savory and spicy snacks has been reported [21,22].

Percent acceptance of the ancient whole grain glutenfree Quinoa-Peanut meal-Beets snacks is given in Figure 3. Acceptance of QPB-Onion, QPB-Garlic and QPB snacks was similar (78-88\%) and significantly higher than QPB-Ginger (65\%) snacks. Except for QPB-Ginger acceptance by in-house seventy seven tasters for the whole grain gluten-free snacks tested was very encouraging. 
Nutrition \& Food Science International Journal

Table 4: Water Activity (Aw), True Density ( $\rho t)$, Bulk Density ( $\rho b)$, Porosity and Expansion of Quinoa-Peanut meal-Beets (QPB) Whole Grain, Gluten-Free, and High Protein Snacks.

\begin{tabular}{|c|c|c|c|c|c|}
\hline Snack & Aw & Pt & Pb & Porosity & Expansion \\
\hline QPB & $0.46 \pm 0.01^{\mathrm{ab}}$ & $1.36 \pm 0.01^{\mathrm{b}}$ & $0.49 \pm 0.04^{\mathrm{a}}$ & $0.64 \pm 0.01^{\mathrm{a}}$ & $2.8 \pm 0.09^{\mathrm{a}}$ \\
\hline QPB-Onion & $0.43 \pm 0.01^{\mathrm{b}}$ & $1.37 \pm 0.01^{\mathrm{a}}$ & $0.55 \pm 0.02^{\mathrm{a}}$ & $0.60 \pm 0.01^{\mathrm{b}}$ & $2.5 \pm 0.03^{\mathrm{b}}$ \\
\hline QPB-Garlic & $0.45 \pm 0.01^{\mathrm{ab}}$ & $1.34 \pm 0.01^{\mathrm{c}}$ & $0.50 \pm 0.03^{\mathrm{a}}$ & $0.62 \pm 0.01^{\mathrm{ab}}$ & $2.7 \pm 0.05^{\mathrm{ab}}$ \\
\hline QPB-Ginger $^{\mathrm{b}}$ & $0.47 \pm 0.01^{\mathrm{a}}$ & $1.33 \pm 0.01^{\mathrm{d}}$ & $0.48 \pm 0.04^{\mathrm{a}}$ & $0.64 \pm 0.01^{\mathrm{a}}$ & $2.8 \pm 0.09^{\mathrm{a}}$ \\
\hline
\end{tabular}

aValues (mean \pm SEM) within columns with different superscript letters differ significantly $(p \leq 0.05), n=77$..

Water activity $(\mathrm{Aw})$ was measured $(\mathrm{N}=3)$ at $25.01 \pm 0.02^{\circ} \mathrm{C}$ by AquaLab dew point water activity meter 4TE (Decagon Devices, Inc., Pullman, WA).

True density ( $\rho$ t) was determined ( $\mathrm{N}=5$ ) by AccPyc II 1340 gas pycnometer (Micromeritics Instrument Co., Norcross, $\mathrm{GA}$ ) at $21.4 \pm 0.4^{\circ} \mathrm{C}$.

The bulk density $(\rho b)$ of each sample was measured $(\mathrm{N}=3)$ by Ottawa Sand volume displacement by about $10 \mathrm{~g}$ of sample in triplicate after 15-5-5 minutes shaking in a jar of 202 cc volume.

Porosity was calculated using the equation [Porosity $=1-(\rho b / \rho t)]$.

Expansion was calculated using the equation [Expansion $=(\rho t / \rho b)]$

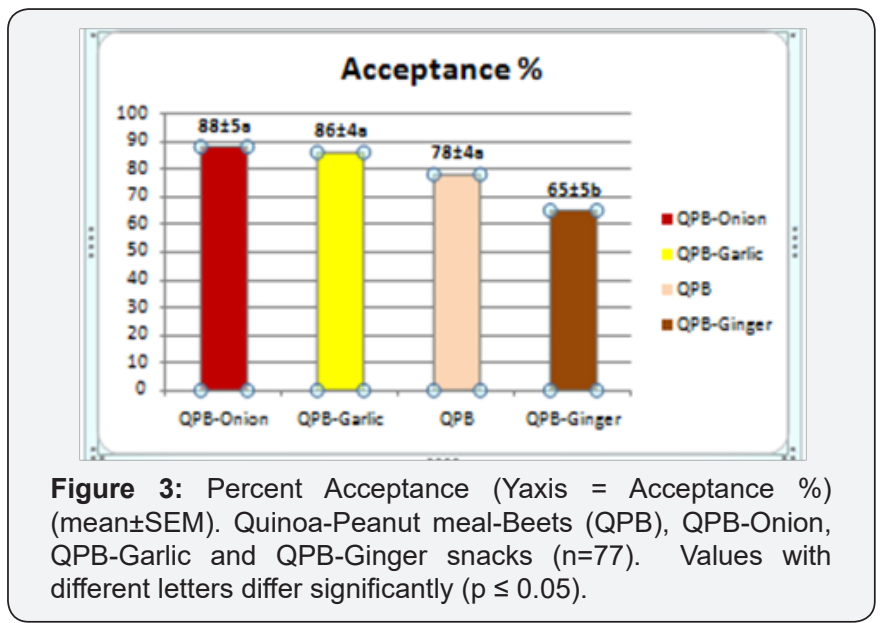

Water activity (Aw) of QPB-Onion snacks was significantly lower than QPB-Ginger snacks Table 4. Aw of the snacks tested ranged from 0.43-0.47. Data suggests that all the snacks tested were crispy with good anti-microbial stability. In the study reported herein Aw values were similar to those reported for Carrots snacks with Aw of 0.44 with desired crispness and antimicrobial stability [23-25]. True density of the four kinds of snacks tested was significantly different from each other. True density $(\rho t)$ values for snacks were $Q P B-O$ nion $>Q P B>Q P B-$ Garlic $>Q P B-G i n g e r$. Bulk density $(\rho b)$ values of the snacks tested were similar and the values ranged 0.48-0.55. Porosity and expansion values of the QPB and QPB-Ginger snacks were similar and significantly higher than QPOB-Onion snacks. Expansion values for the snacks tested ranged 2.5-2.8 with porosity of 60-64\% suggest that these snacks were quite fluffy, brittle. Previously lower Aw (0.2-0.3), higher expansion 4.1-4.5 and porosity of 0.76-0.78 have been reported for gluten-free vegetable snacks without added protein have been reported [22]. Suggesting that addition of protein resulted in higher Aw and lower expansion and porosity is the study reported herein. Data suggest that the snacks tested would be filling and give reasonable presentation in their commercial packaging.

Composition of ancient whole grain, gluten-free, high protein, vegetable snacks is given in Table 5 . The goal was use peanut meal, a low value farm byproduct, to increase the protein content of the snacks to $25 \%$. Protein level of the snacks tested ranged from 24-28\%. This object was successfully attained except for QPB-Ginger snacks where it was $0.55 \%$ lower than desired. Fat content of the formulated snacks was $8-11 \%$. In the formulation of these snacks only $1.2 \%$ salt was added Table 2 . The mineral content of the snacks tested ranged $5.03-5.51 \%$ suggesting that the snacks were low sodium and good source of other essential minerals. The snacks tested were more than $50 \%$ lower in fat and salt than some of the whole grain vegetable snacks available in the market.

Table 5: Composition of Quinoa-Peanut meal-Beets (QPB) Whole Grain, Gluten-Free, and High Protein

\begin{tabular}{|c|c|c|c|c|c|}
\hline Snack & Protein & Fat & Minerals & Carbohydrates & DM \\
\hline QPB & $28.05 \pm 1.15$ & $8.00 \pm 0.02$ & $5.51 \pm 0.01$ & 50.76 \\
\hline QPB-Onion & $27.21 \pm 1.58$ & $11.19 \pm 0.03$ & $5.03 \pm 0.01$ & 49.11 & $92.54 \pm 0.02$ \\
\hline QPB-Garlic & $26.35 \pm 1.70$ & $8.14 \pm 0.09$ & $5.34 \pm 0.01$ & 53.16 & $92.99 \pm 0.04$ \\
\hline QPB-Ginger & $24.45 \pm 1.62$ & $10.85 \pm 0.04$ & $5.41 \pm 0.03$ & 51.83 & $92.54 \pm 0.02$ \\
\hline
\end{tabular}

Nitrogen to protein factors used was 6.25. Dry matter, DM. Carbohydrate $=[$ Dry Matter $-($ Protein + Fat + Ash $)]$. Samples were analyzed in triplicates. Values are mean \pm SEM, $(n=3)$. 


\section{Conclusion}

Protein content of the snacks tested ranged from 24.5$28 \%$. It has added value to peanut meal a farm byproduct mainly used as animal feed. Acceptance of QPB-Onion, QPBGarlic, QPB snacks was similar (78-88\%) and significantly higher than QPB-Ginger (65\%) snacks. Except for QPB-Ginger snacks these acceptance values are very encouraging. These snacks are formulated using only 3-4 ingredients and could be made in any house hold on counter top appliances and/or in commercial productions. The water activity of these snacks ranged 0.43-0.47 suggesting that the snacks were crispy with good antimicrobial stability. Expansion of the cooked snacks was 2.5-2.8 with $60-64 \%$ porosity suggesting that these snacks are light and brittle and would be quite filling with desirable presentation in commercial packaging. The snacks tested were more than $50 \%$ lower in fat and salt than some of the whole grain vegetable snacks available in the market. These whole grain gluten-free high protein vegetable healthy nutritious tasty snacks offer choice for vegetarians as well as individuals sensitive to gluten.

\section{References}

1. Prepared Foods (2016).

2. USDA (2015) Nutrition Policy and Promotion; Dietary Guidelines for Americans.

3. Whole Grain Council (2009).

4. Halnaes A, Kyro C, Anderson I, Lacoppidan S, Overvad K, et al. (2016) Intake of whole grains is associated with lower risk of myocardial infarction: the Danish Diet, Cancer and Health Cohort. Am J Clin Nutr 103(4): 999-1007.

5. Lebwohl B, Ludvigsson JF, Green PH (2015) Celiac disease and nonceliac gluten sensitivity B M J (Review) 351: h4347.

6. Food and Drug Administration defines gluten-free (2014).

7. Hollon JR, Cureton PA, Martin ML, Puppa EL, Fasano A (2013) Trace gluten contamination may play a role in mucosal and clinical recovery in a subgroup of diet-adherent non-responsive celiac disease patients BMC Gastroenterol 13: 40.

8. Mother-Jones (2017) No, Feeding Hungry Kids and Seniors Isn't a Waste of Money.

9. Food and Agricultural Organization of the United Nations (2012).
10. Kahlon TS, Chapman MH, Smith GE (2007) In vitro binding of bile acids by okra, beets, asparagus, eggplant, turnips, green beans, carrots, and cauliflower. Food Chem 103: 676-680.

11. Slimestad R, Fossen T, Vågen IM (2007) Onions: A source of unique dietary flavonoids. J Agr \& Food Chem 55(25): 10067-10080.

12. Rahman K (2007) Effects of garlic on platelet biochemistry and physiology. Mol Nutr Food Res 51: 1335-1344.

13. Guercio V, Turati F, La Vecchia C, Galeone C, Tavani A (2016) Allium vegetables and upper aerodigestive tract cancers: a meta-analysis of observational studies. Molecular Nutrition \& Food Res 60(1): 212-222.

14. Thomson M, Al-Qattan KK, Al-Sawan SM, Alnaqeeb MA, Khan I, et al. (2002) The use of ginger (Zingiber officinale Rosc.) as a potential anti- inflammatory and antithrombotic agent. Prostaglandins Leukot. Essent. Fatty Acids 67(6): 475-478.

15. Akinyemi AJ, Thomé GR, Morsch VM, Bottari NB, Baldissarelli J, et al. (2016) Dietary Supplementation of Ginger and Turmeric Rhizomes Modulates Platelets Ectonucleotidase and Adenosine Deaminase Activities in Normotensive and Hypertensive Rats. Phytotherpy Res 30(7): 1156-1163.

16. Klein C, Sato T, Meguid MM, Miyata G (2000) From food to nutritional support to specific nutraceuticals: A journey across time in the treatment of disease. J Gastroenterol Suppl 12: 1-6.

17. AOAC (2000) Official Methods of Analysis of the Association of Official Analytical Chemists, $\left(17^{\text {th }}\right.$ edn), The Association: Arlington, VA, methods 985.29 and 990.03 .

18. AOAC (1990) Official Methods of Analysis of the Association of Official Analytical Chemists, $\left(15^{\text {th }}\right.$ edn). The Association: Arlington, VA., methods 935.29 and 942.05 .

19. Kahlon TS, Milczarek RR, Chiu MM (2013) Whole Grain Gluten-free Egg-free Pasta. Cereal Foods World 58(1): 4-7.

20. Kahlon TS, Milczarek RR, Chiu MM (2014) Whole Grain Gluten-free Egg-free High Protein Pasta. Vegetos 26: 65-71.

21. Kahlon TS, Avena-Bustillos RJ, Chiu MM, Hidalgo MB (2014) Whole Grain Gluten-Free Vegetable Savory Snacks. J Food Res 3(5): 31-37.

22. Kahlon TS, Avena-Bustillos RJ, Chiu MM, Hidalgo MB (2015) Whole Grain Gluten-Free Vegetable Spicy Snacks. J Food Res 4(5): 57-63.

23. Adams MR, Moss MO (1997) Food microbiology, Cambridge: The Royal Society of Chemistry.

24. Dueik V, Marzullo C, Bouchon P (2013) Effect of vacuum inclusion on the quality and the sensory attributes of carrot snacks. Food Sci Technol 50(1): 361-365.

25. Water Activity in Foods: Fundamentals and Applications (2007) In: Barbosa-Canovas GV, et al. (Eds.), Wiley-Blackwell Press.

\section{Your next submission with Juniper Publishers will reach you the below assets}

- Quality Editorial service

- Swift Peer Review

- Reprints availability

- E-prints Service

- Manuscript Podcast for convenient understanding

- Global attainment for your research

- Manuscript accessibility in different formats ( Pdf, E-pub, Full Text, Audio)

- Unceasing customer service

Track the below URL for one-step submission https://juniperpublishers.com/online-submission.php 